\title{
Research Paper \\ The Status of Potentially Inappropriate Medication Prescription by General Physicians for The Elderly in Tabriz (Iran) According to Beers Criteria
}

\author{
Tahereh Eteraf Oskouei ${ }^{1}$ (C), Elaheh Vatankhah ${ }^{1} \mathbb{1}^{\circ},{ }^{*}$ Moslem Najafi $^{2}$ (B)
}

1. Department of Pharmacology and Toxicology, Faculty of Pharmacy, Tabriz University of Medical Sciences, Tabriz, Iran.

2. Medical Education Research Center, Health Management and Safety Promotion Research Institute, Tabriz University of Medical Sciences, Tabriz, Iran.

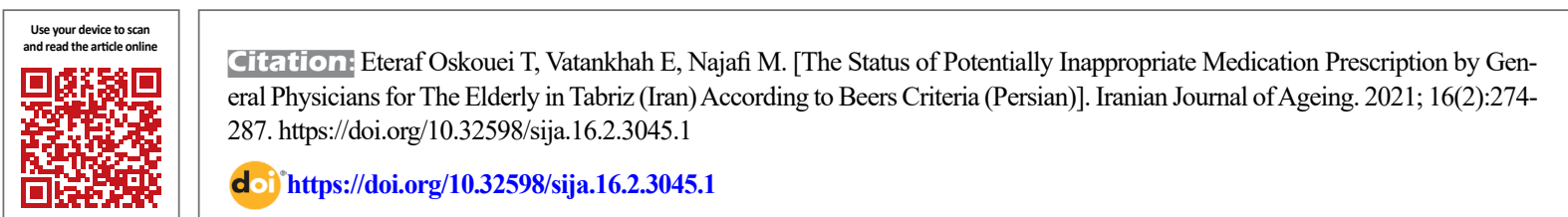

(c) (i) (5)

Received: 03 Sep 2020 Accepted: 17 Nov 2020 Available Online: $01 \mathrm{Jul} 2021$

Key words: Potentially inappropriate medications, Elderly, General physicians, Beers criteria, Tabriz

\section{ABSTRACT}

Objectives Prescribing medication for the elderly is an important health issue. This study aimed to investigate the status of Potentially Inappropriate Medications (PIMs) prescription for the elderly by general physicians in Tabriz, Iran.

Methods \& Materials This descriptive cross-sectional study was conducted in 2019 on the prescriptions of general physicians for the elderly in Tabriz, Iran. After initial screening of 16842 prescriptions belonged to two insurance systems (social security insurance and health insurance), 1500 prescriptions were selected. The Beers criteria 2019 was used to assess PIM prescription status and their alternatives were suggested if needed. Data were expressed as Mean \pm Standard Deviation (SD) and No. (\%), and analyzed in SPSS v. 21 software. The Mann-Whitney $U$ test was used to examine the differences in the study factors between the prescriptions related to two insurance systems. The relationship between patients' age and PIM prescription was examined using Spearman correlation test.

Results The Mean \pm SD number of drugs in each prescription was $3.93 \pm 1.5$ and the Mean \pm SD number of prescribed PIMs was $0.74 \pm 0.8$. Polypharmacy was observed in $32.6 \%$ of the prescriptions, and $53.9 \%$ had at least one PIM. Non-steroidal anti-inflammatory drugs, antihistamines and benzodiazepines were the most PIM groups (45.6, 10.9 and 10.6\%, respectively). The most common prescribed PIMs were Adult cold, Diclofenac, Glibenclamide, Ketorolac and Diphenhydramine. Number of PIMs in each prescription did were not statistically different between the prescriptions related to two insurance systems $(P=0.343)$. Moreover, patients' age and gender had no significant relationship with PIM prescription ( $\mathrm{P}=0.312$ and $\mathrm{P}=0.660$, respectively).

Conclusion About $54 \%$ of the prescriptions issued by general physicians for the elderly in Tabriz contain PIMs, and polypharmacy is present in about one third of the prescriptions. In order to improve this condition, it is necessary to hold appropriate training and continuous retraining courses in geriatric medicine for general physicians. Supervisions by the Iranian Ministry of Health and Iranian Medical Council can also play an important role in improving the current situation.

\section{* Corresponding Author:}

Moslem Najafi, Pharm D.

Address: Medical Education Research Center, Health Management and Safety Promotion Research Institute, Tabriz University of Medical Sciences, Tabriz, Iran.

Tel: +98 (41) 33372250

E-mail: najafim@tbzmed.ac.ir 


\section{Extended Abstract}

\section{Introduction}

A

ccording to the United Nations, the number of older people in the world is projected to double and reach 2.1 billion by 2050 [1]. In Iran, the population over 60 years old in 2006 was about $7 \%$ and is estimated to reach about $22 \%$ in 2036 [3]. In old age, in addition to the increase in the burden of disease, the time of using health services, and health costs $[4,5]$, polypharmacy is common and can significantly increase the risk of side effects, drug interactions, medication errors, hospitalization and even death [8-10]. In many countries, the Beers criteria is used to estimate the prescription of Potentially Inappropriate Medications (PIMs) in the elderly [15]. The purpose of this study was to investigate the status of PIM prescription for the elderly according to Beers criteria in Tabriz, Iran.

\section{Methods}

This is a descriptive cross-sectional study. The number of samples was obtained 1421 using Cochran's formula and considering a $95 \%$ confidence interval, $\mathrm{Z}(1-\alpha / 2)=1.96$, $\alpha=0.05, \mathrm{P}=1-\mathrm{P}=0.5$, and $\mathrm{d}=0.026$. For more accuracy, it was set to 1500 . By referring to the social security and health insurance organizations of East Azarbaijan province, the prescriptions belonging to people aged 65 years and older who were prescribed by general physicians in Tabriz city in winter and spring 2019 were collected. First, the prescriptions were screened and if they belonged to the target group, they were separated. Finally, out of 16,842 prescriptions, 1500 were selected. Also, in order to achieve more generalizability of the results, the number of selected prescriptions from each physician was determined up to 10-15. In addition to general patient information (type of insurance, age and gender), the name of prescribed medications, drug group, number of items in each prescription, and the average number of prescription items were recorded. Then, according to the Beers criteria 2019, the name and number of prescribed PIMs were determined and if there were appropriate medications, their alternatives were sug-

Table 1. All PIMs in the study prescriptions

\begin{tabular}{|c|c|}
\hline Medication Group & PIMs \\
\hline NSAIDs & Indomethacin, Piroxicam, Mefenamic acid, Meloxicam, Ibuprofen, Diclofenac, Naproxen, Ketorolac, Adult Cold \\
\hline $\begin{array}{l}\text { Anxiolytics (Benzodiaz- } \\
\text { epines) }\end{array}$ & Alprazolam, Clonazepam, Chlordiazepoxide, Lorazepam, Oxazepam, Diazepam \\
\hline Anti-tussives & Expectorant \\
\hline Anti-Spasmodics & Dicyclomine, Clidinium-C, Hyoscine \\
\hline Antidepressants & Nortriptyline, Amitriptyline, Imipramine, Doxepin \\
\hline Gastrointestinal drugs & Metoclopramide \\
\hline Antihistamines & Hydroxyzine, Chlorpheniramine, Diphenhydramine, Clemastine, Cyproheptadine, Dimenhydrinate, Promethazine \\
\hline Muscle relaxants & Methocarbamol \\
\hline Antidiabetics & Glibenclamide \\
\hline Anti-Hypertensives & Prazosin, Clonidine \\
\hline Sedative/Hypnotics & Zolpidem \\
\hline Antiparkinson drugs & Trihexyphenidyl \\
\hline Cardiac drugs & Digoxin \\
\hline Anti-convulsants & Phenobarbital \\
\hline Antipsychotics & Trifluoperazine, Quetiapine \\
\hline
\end{tabular}


gested based on valid scientific sources [14, 28, 29]. Data were expressed as Mean \pm Standard Deviation (SD) and No. $(\%)$ and were analyzed in SPSS v. 21 software. First, the distribution of PIM prescription data was evaluated using Kolmogorov-Smirnov test and due to their abnormal distribution, Mann-Whitney U test was used for statistical analysis. Spearman correlation test was used to examine the relationship between patients' age and PIM prescription. $\mathrm{P}<$ 0.05 was considered as the significance level.

\section{Results}

Among 1500 prescriptions, 571(38.1\%) was related to health insurance and $929(61.9 \%)$ for social security insurance; 876(58.4\%) belonged to older women and $624(41.61 \%)$ belonged to older men and the Mean \pm SD age of all patients was $73.31 \pm 6.8$ years. On average, $3.93 \pm 1.5$ medications were prescribed for each participant; $4 \%$ contained one drug, $12.8 \%$ contained 2 drugs, $25.5 \%$ contained 3 drugs, $25.1 \%$ contained 4 drugs, $16.4 \%$ contained 5 drugs and $16.2 \%$ contained 6 and more drugs. Therefore, according to the definition of polypharmacy (using 5 drugs or more), there was polypharmacy in $32.6 \%$ of the studied prescriptions. Moreover, 809 prescriptions (53.9\%) contained PIMs according to Beers criteria; $37.8 \%$ of prescriptions had one PIM, $13.1 \%$ two PIMs and $3 \%$ three or four PIMs. All prescribed PIMs were classified into 15 medication groups, which are listed in Table 1. Among these groups, Nonsteroidal Anti-Inflammatory Drugs (NSAIDs) with a frequency of $45.6 \%$, antihistamines $(10.9 \%)$, benzodiazepines (10.6\%), anti-diabetic drugs $(8.9 \%)$, antispasmodics $(5.1 \%)$, antidepressants $(4.1 \%)$, antitussive drugs $(3.7 \%)$ and gastrointestinal drugs $(2.4 \%)$ were more common. Moreover, the most common prescribed PIMs were: Adult cold (14.5\%), Diclofenac (10.4\%), Glibenclamide (8.9\%), Ketorolac (6.9\%), Diphenhydramine (4.7\%), Ibuprofen (4.3\%), Clonazepam (3.8\%), Indomethacin (3.7\%), Alprazolam (3.4\%), and Piroxicam (2.8\%).

Using Mann-Whitney U test, comparison of the mean number of prescribed PIMs in each prescription did not show a statistically significant difference between those related to health insurance and social security $(\mathrm{P}=0.343)$. The relationship between age and PIM prescription was assessed using Spearman correlation test and the effect of gender on PIM prescription was evaluated using MannWhitney $\mathrm{U}$ test for which the $\mathrm{P}$ values were 0.312 and 0.660 , respectively. Therefore, the variables of age, gender and type of insurance had no significant relationship with the frequency of PIM prescription.

\section{Discussion and Conclusion}

According to the obtained data, about $54 \%$ of prescriptions issued by general physicians for the elderly in Tabriz in 2019 contained PIMs, and polypharmacy was observed in $32.6 \%$ of prescriptions. Due to the high polypharmacy and the rate of PIM prescriptions for the elderly in this study and previous similar studies conducted in Iran, which imposes high financial costs, drug side effects, increased hospitalization and even death, there is a need to develop and implement scientific criteria and guidelines for prescribing medications to the elderly, raising the awareness of general physicians and increasing the supervision of relevant authorities. Moreover, due to the increase in the aged population and the spread of chronic and non-communicable diseases, it is necessary to pay serious attention to the issue of geriatrics and pharmacotherapy of the elderly in medical universities and medical retraining courses. Furthermore, holding supplementary geriatric medicine courses to train employed general physicians can be helpful.

\section{Ethical Considerations}

\section{Compliance with ethical guidelines}

This study has an ethical approval (Code: IR.TBZMED. REC.1397.1033) obtained from the Research Ethics Committee of Tabriz University of Medical Sciences. All information on the prescriptions examined in this study was kept confidential.

\section{Funding}

This study was extracted from a Pharm D. dissertation of the second author at the Department of Pharmacology and Toxicology, Faculty of Pharmacy, Tabriz University of Medical Sciences, Tabriz.

\section{Authors' contributions}

All authors contributed equally in preparing this article.

Conflicts of interest

The authors declare no conflict of interest

\section{Acknowledgements}

The authors would like to thank the officials of the Health Insurance Organization and the Social Security Insurance Organization of East Azerbaijan Province for their cooperation. 


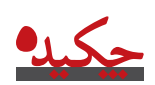

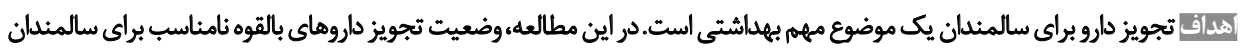
در نسخ يزٔشكان عمومى بروسى شدائ

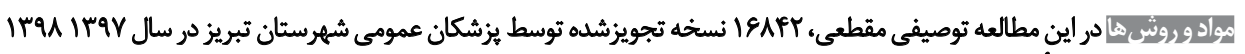

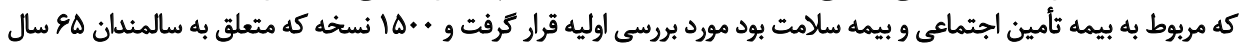

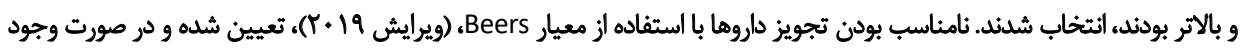

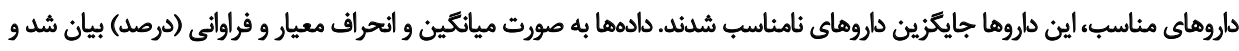

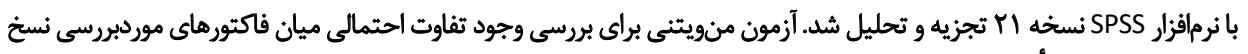

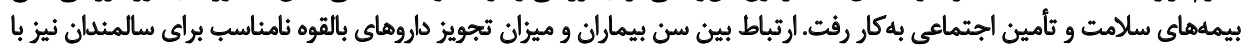

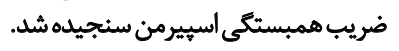

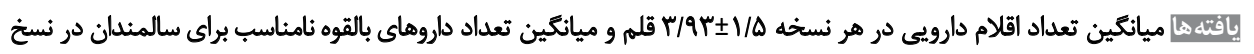

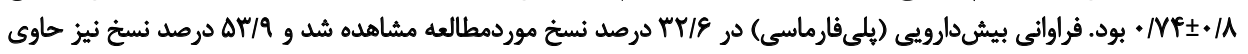

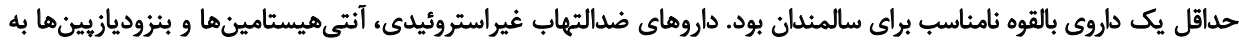

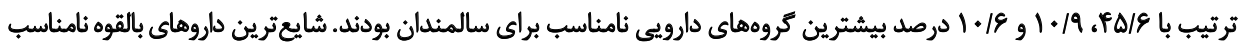

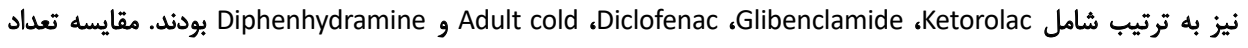

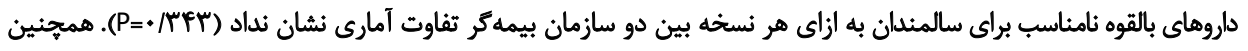

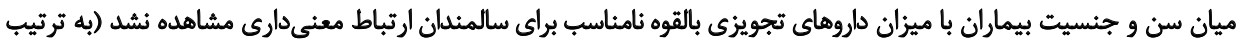
(P=⿰㇒⿻二丨丿

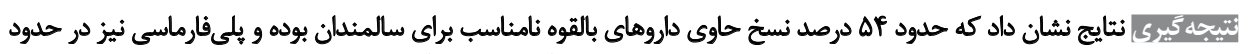

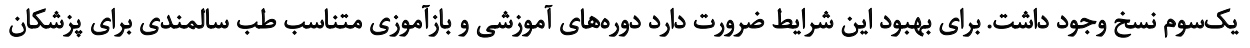

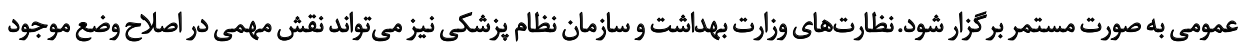
ايفاكئد.

\author{
تاريخ دريافت: تا شهريور

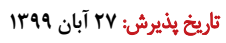

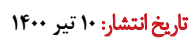

كليدواثرها: داروهاى بالقوه نامناسب،

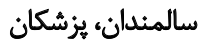

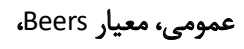
تبريز
\end{abstract}

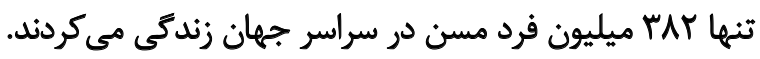

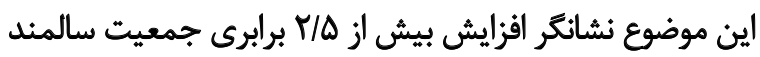

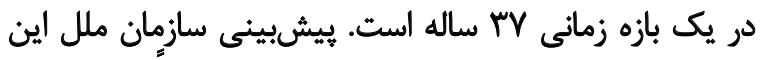

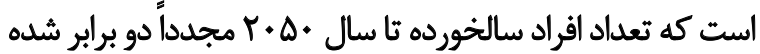

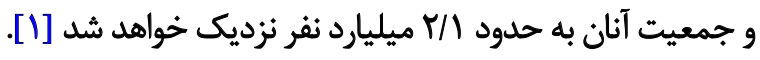

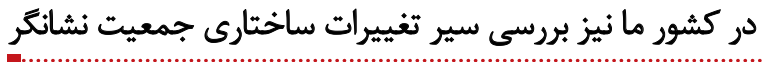

سالمندى ييشرونده جمعيت جهان يكى از بارزترين يديدهاي

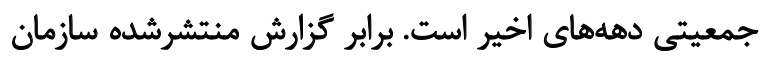

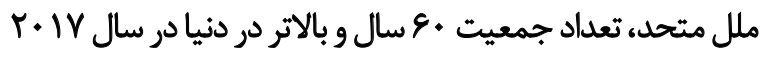

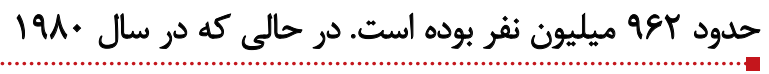

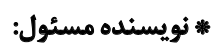

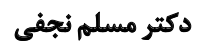
نشانى: تبريز، دانشكاه علوم يزشكى تبريزّ، يرؤشككده مديريت سلامت و ارتقاى ايمنى، مركز تحقيقات آموزش يزشكى.

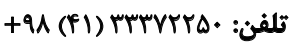
najafim@tbzmed.ac.ir بست الكترونيكى 
حدود دوسوم آنان نيز به مراقبتهاي يزشكى نياز يبيدا خواهند كرد.

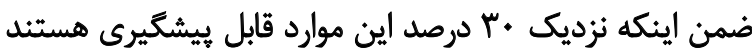

معيار Beers براى اولينبار در سال 1991 ميلادى توسط دكتر

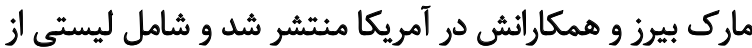

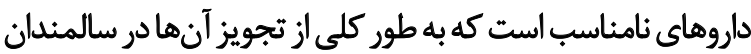

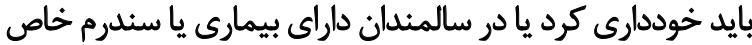

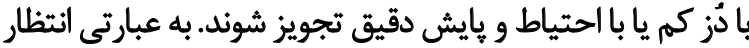

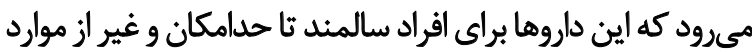

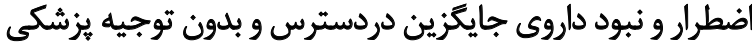

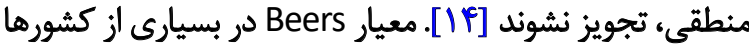

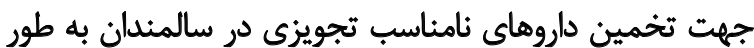

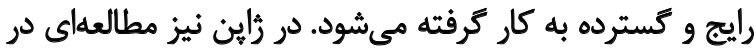

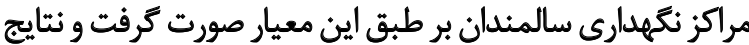



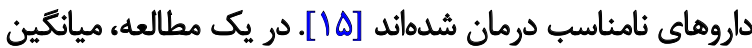

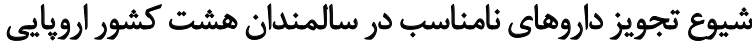

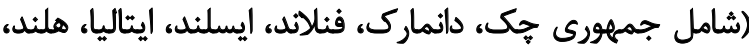

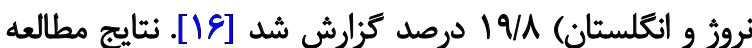

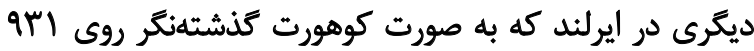

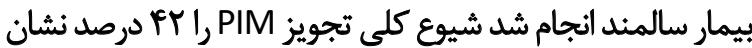

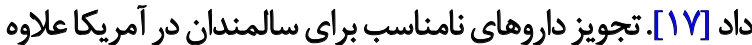

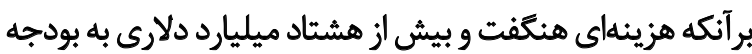



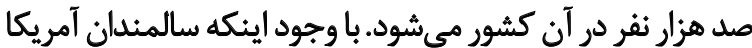

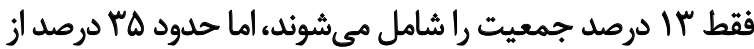

كل هزينههاى دارويى به اين تروه سنى تعلق دارد [N/N].

نتايج يك مطالعه در آفريقاى جنوبى روى بيش از باء ـا هزار بيمار سالمند 90 سال و بالاتر نشان داد كه بران براى

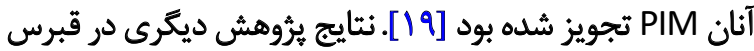

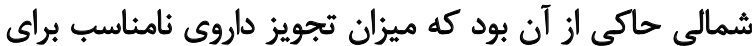

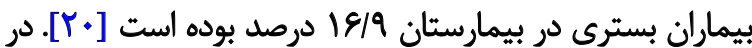

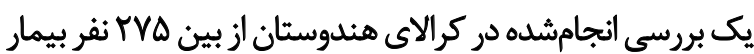

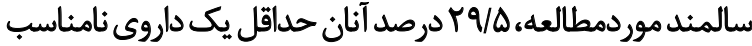



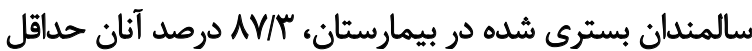

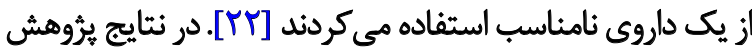

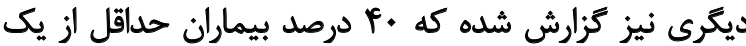

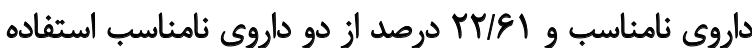

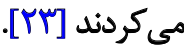

در يك مطالعه مقطعى در بيمارستان رسول اكرم (ص) شهر

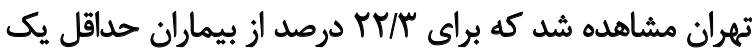

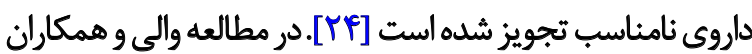

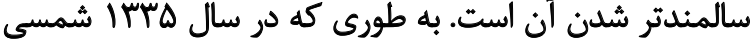

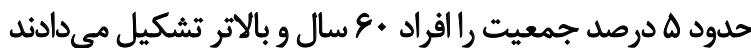

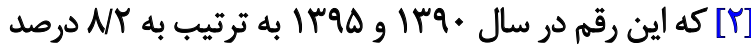

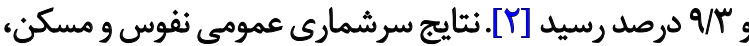

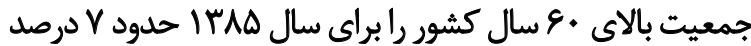

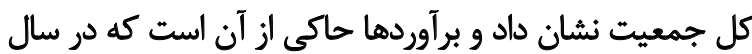

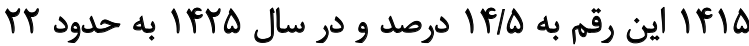

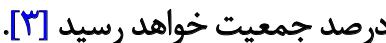

در دوران سالمندى، هزينههاى درمانى به ميزان قابل توجهى ئري

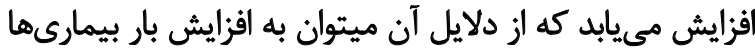

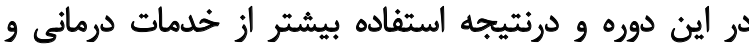

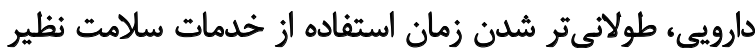

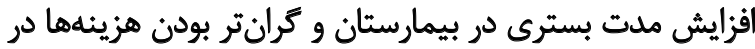

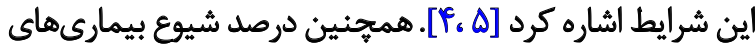

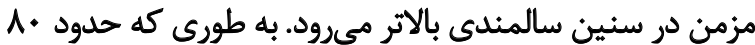

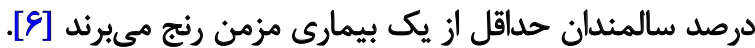

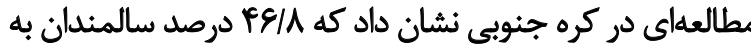

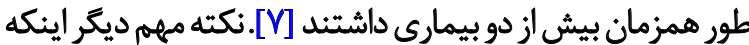

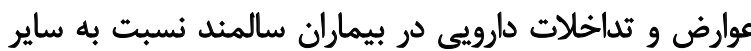

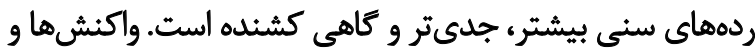

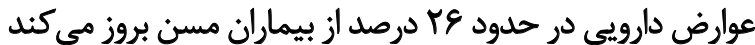

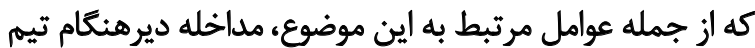

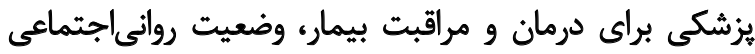

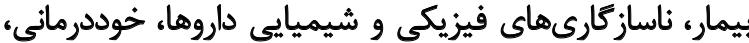

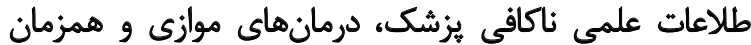

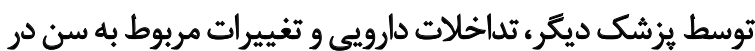

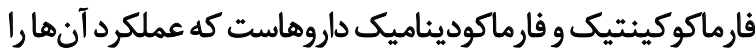
در بيماران سالمند تغيير مي دهد [A،

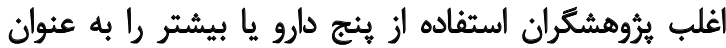

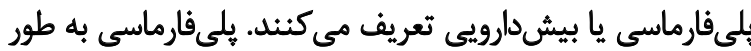

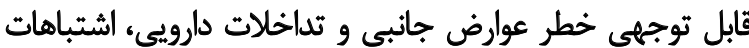

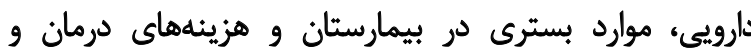

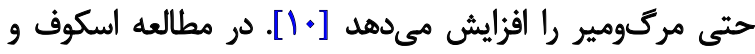

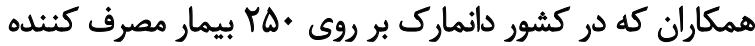

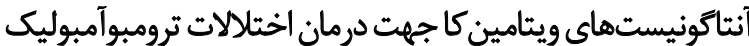

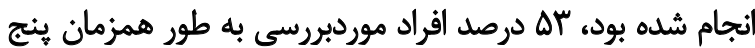

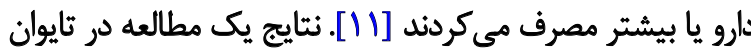

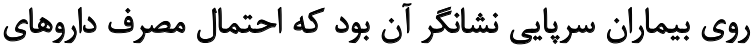

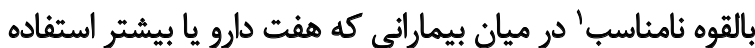

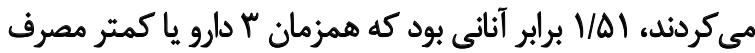

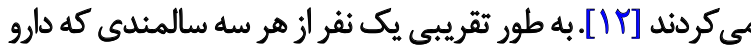

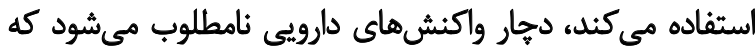

1. Potentially Inappropriate Medication (PIM) 


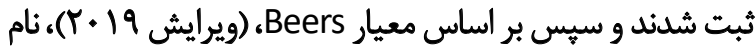

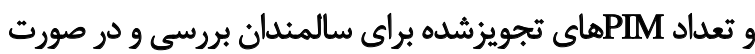
وجود داروهاى مناسب، اين داروها، به عنوان جايكزين دارئ داروهاى براي

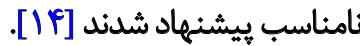

$$
\text { ووش آمارى }
$$

دادههاى جمعآورىشده برحسب مورد به صورث انحراف

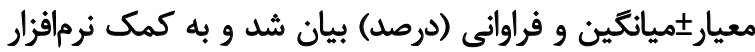



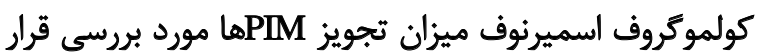

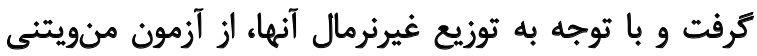

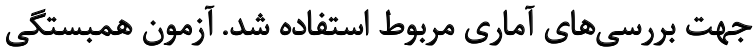

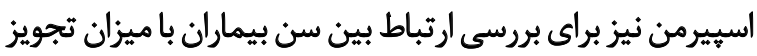

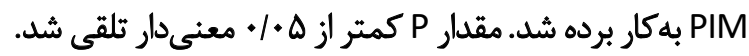

Latị

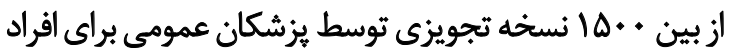

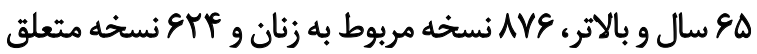

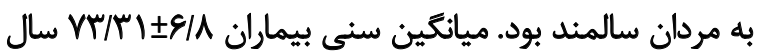

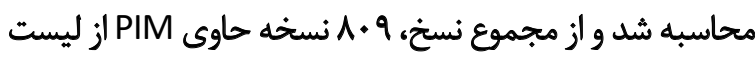

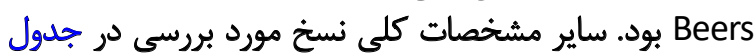
شماره ال آورده شده است.

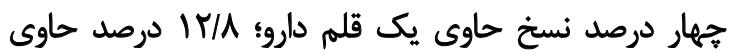
دو قلم؛

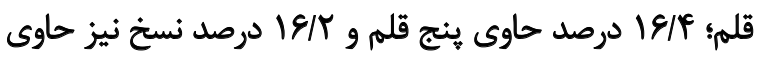

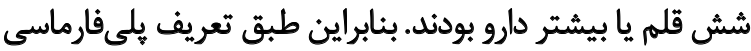

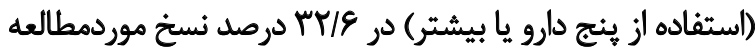

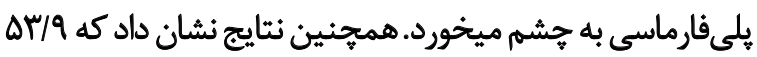

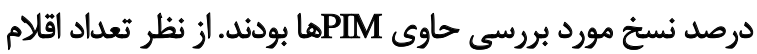

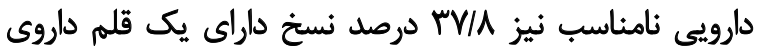

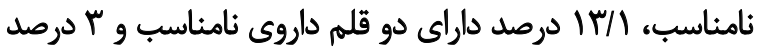

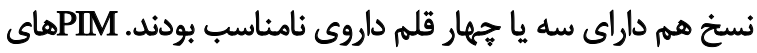

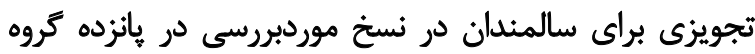

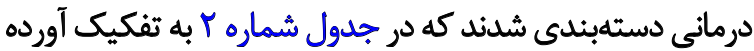
شده است. درسيت

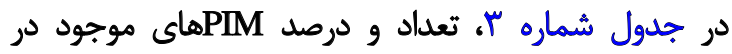

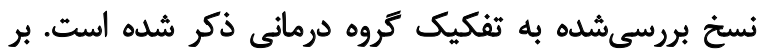

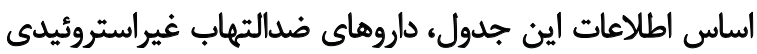
(NSAIDS) Pاى تجويزى براي سالمندان برمبناى ليست Beers بونئد

در تصوير شماره ا نيز فراوائى (درصد) شايعترين PIM

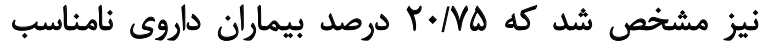

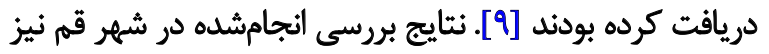

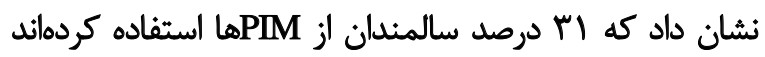

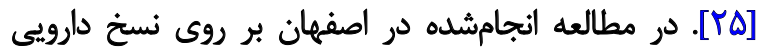

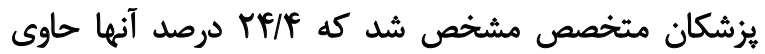

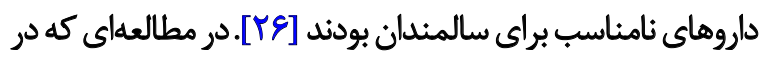

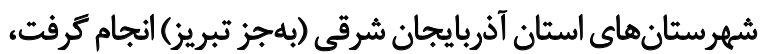

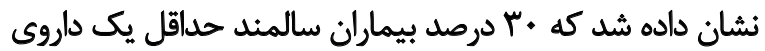

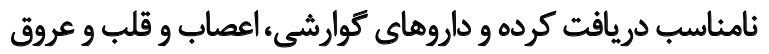

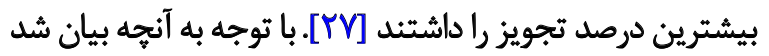

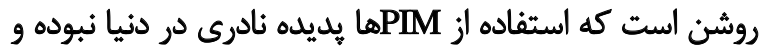
با وجود اينكه شواهدى از نتايج نامطلوب استفاده از اين داروها داردا

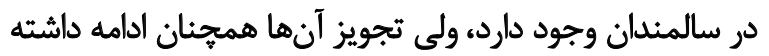

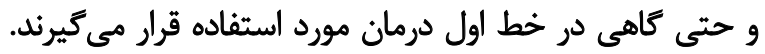

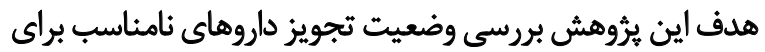

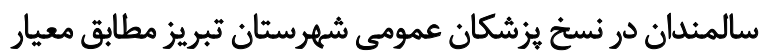
Beers

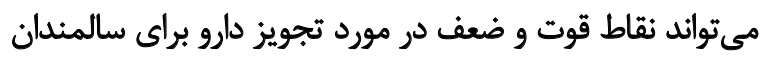

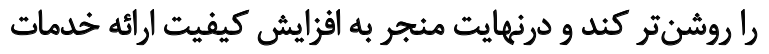
سلامت به سالمندان و كاهش هزينه درنهاى جامعنه شود.

$$
\text { ووش مطأله }
$$

اين مطالعه به صورت توصيفى مقطعى طراحى واجرا شد. تعداد

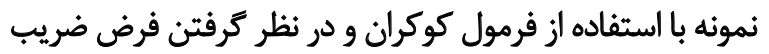
$Z(\mid-\alpha / T)=1 / 9$ ا

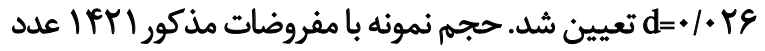

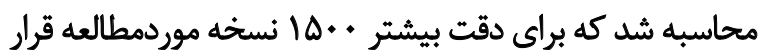

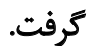
براي تهيه نسخ،طى معرفي نامهاي به سازمانهاي بيمه تأمين

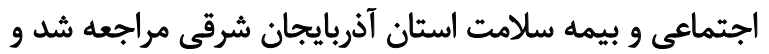

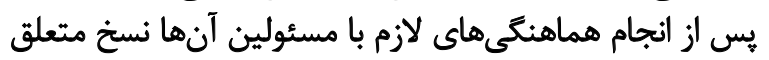

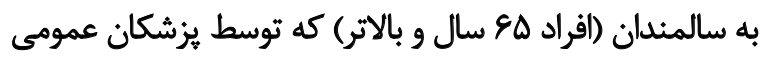

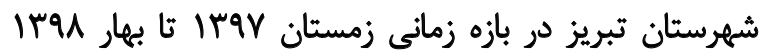

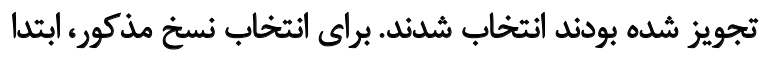

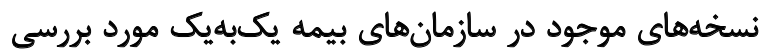

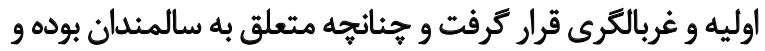

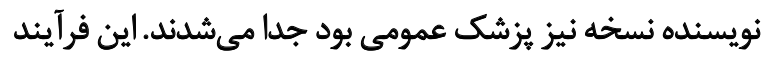

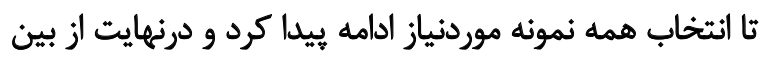
أlANFr

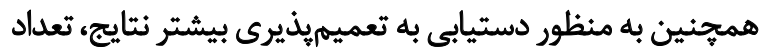

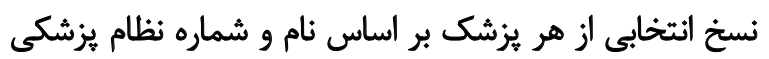

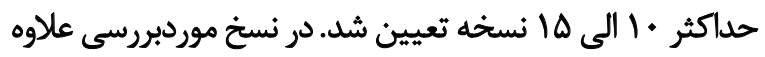

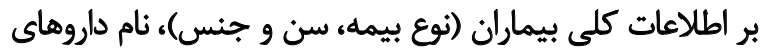

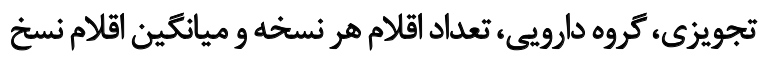


جدول ا. اطلاعات كلى نسخ موردمطالعه

\begin{tabular}{|c|c|}
\hline مقدار & شاخص موردبررسى در نسخ \\
\hline IFAFT & تعداد كل نسخ موردبررسى اوليه و غربالكرى شده \\
\hline $10 \cdots(N 9)$ & تعداد نسخ يزشكان عمومى تجويزشده براى سالمندان (درصد از كل نسخ) \\
\hline$\Delta V(Y N)$ & تعلاد نسخخ مريوط به بيمه سلامت (درصد از كل نسخ مريوط به سالمندان) \\
\hline qหq (E)/q) & تعلاد نسخ مربوط به بيمه تأمين اجتماعي (درصد از كل نسخ مربوط به سالمندان) \\
\hline$\Gamma / q \Psi \pm V / \Delta$ & هيانكيندانحراف معيار تعداد اقلام دارويى در هر نسخه به طور كلى \\
\hline $4 / 1 \cdot \pm 1 / \Delta 9$ & ميانكين ثانحراق معيار تعلداد اقلام دارويع در هر نسخه براى نسخ بيمه سلامت \\
\hline$r / A r \pm 1 / f q$ & هيانكين||نحراف هعيار تعداد اقلام دارويى در هر نسخه براي نسخ بيمه تأمين اجتماعى \\
\hline$A V \&(\Delta N F)$ & تعلداد نسخ مربوط به بيماران زن (درصد) \\
\hline $\sin (F / 8)$ & تعلاد نسخ مربوط به بيماران مرد (درصل) \\
\hline$\Lambda \cdot q(\Delta r / q)$ & تعداد نسخ حاوى PIMs (درصد از كل نسخ مربوط به سالمندان) \\
\hline$\cdot / M \pm \cdot / A$ & ميائكين||تحراف هعيار تعداد PIMs در هر نسخخه به طور كلى \\
\hline$\bullet / N^{\omega} \pm / / \Lambda \Delta$ & ميانكين |نانحراف معيار تعلداد PIMs در هر نسخه براى نسخ بيمه سلامت \\
\hline$\cdot / M^{e} \pm . / M 9$ & هيانكين+|نحراف معيار تعداد PIMs در هر نسخه براي نسخ بيمه تأمين اجتماعى \\
\hline
\end{tabular}

جدول Y. داروهاي تجويزى از ليست Beers به تفكيك كروه درماني در نسخ موردمطالعه

داروهاى موجود در نسخهداي موردبررسى

Indomethacin, Piroxicam, Mefenamic acid, Meloxicam, Ibuprofen, Diclofenac, Naproxen, Ketorolac, Adult Cold

Alprazolam, Clonazepam, Chlordiazepoxide, Lorazepam, Oxazepam, Diazepam

\section{Expectorant}

Dicyclomine, Clidinium- $C$, Hyoscine

Nortriptyline, Amitriptyline, Imipramine, Doxepin

$$
\text { Metoclopramide }
$$

Hydroxyzine, Chlorpheniramine, Diphenhydramine, Clemastine, Cyproheptadine, Dimenhydrinate, Promethazine

\section{Methocarbamol \\ Glibenclamide \\ Prazosin, Clonidine

$$
\text { Zolpidem }
$$

Trihexyphenidyl

Digoxin

Phenobarbital

Trifluoperazine, Quetiapine

$$
\text { دسته دارويى }
$$

\section{NSAIDs}

Anxiolytics

(Benzodiazepines)

Anti-tussives

Anti-Spasmodics

Antidepressants

Gastrointestinal Drugs

Antihistamines

Muscle Relaxants

Antidiabetics

Anti-Hypertensives

Sedative/Hypnotics

Antiparkinson Drugs

Cardiac Drugs

Anti-Convulsants

Antipsychotics 
جدول با. فراوائى PIM تجويزى به تفكيك كروهاي دارويى در نسخ موردمطالعه

\begin{tabular}{|c|c|}
\hline فراوانى (درصد) & دسته دارويى \\
\hline$\Delta \cdot r(f \Delta / \varphi)$ & NSAIDs \\
\hline$M(1 . / 9)$ & Antihistamines \\
\hline $117(1 \cdot 19)$ & Benzodiazepines \\
\hline ११(N१) & Antidiabetics \\
\hline$\Delta V(\Delta / 1)$ & Anti-Spasmodics \\
\hline$f \Delta(f / \cdot 1)$ & Antidepressants \\
\hline$P(r / V)$ & Anti-tussives \\
\hline$T V\left(T / T^{e}\right)$ & Gastrointestinal Drugs \\
\hline$M F(T / M)$ & Muscle Relaxants \\
\hline$r F(T / M)$ & Antipsychotics \\
\hline$M F(Y / I)$ & Anti-Hypertensives \\
\hline $9(\cdot(1))$ & Cardiac Drugs \\
\hline$f(\cdot / r q)$ & Sedative/Hypnotics \\
\hline$r(* / r)$ & Antiparkinson Drugs \\
\hline$r(\cdot / r)$ & Anti-Convulsants \\
\hline $11.1(1.0)$ & Total \\
\hline
\end{tabular}

L

اوليه و تفكيك سنى قرار كرفتند، . . 10 نسخه مربوط به افراد

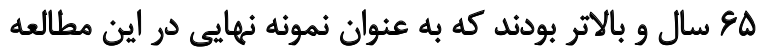

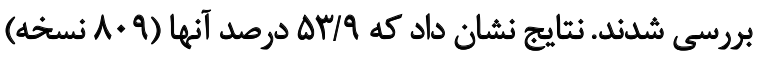

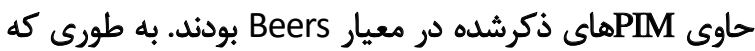

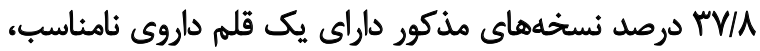

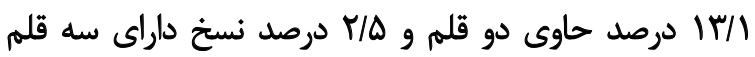

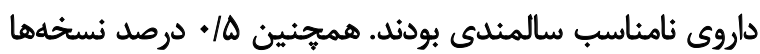



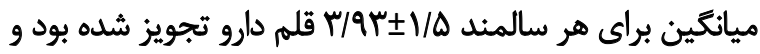

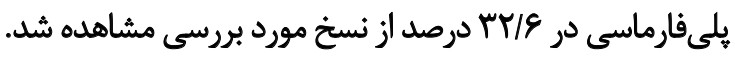
نتايج يك مطالعه انجامشده در شهر كاشان نشان داد كهاد

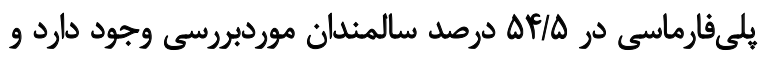

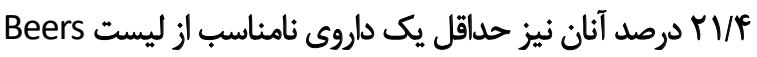

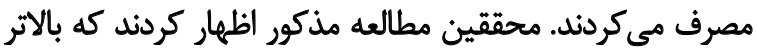

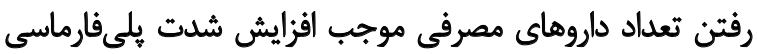

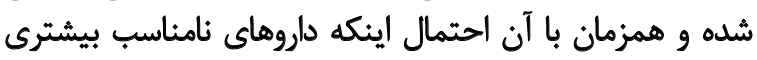

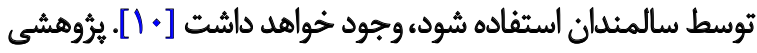

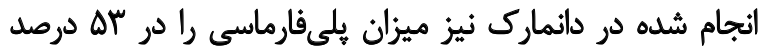

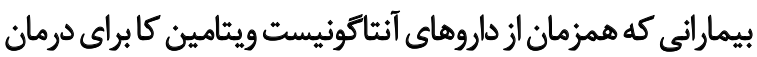

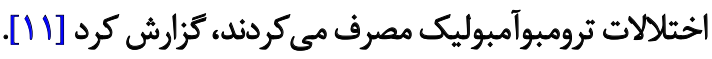
در مطالعه حاضر، بيشترين PIM تجاى تجويز شده براى
تجويزى براى سالمندان نشان داده شده است. با توجه به يافتهها

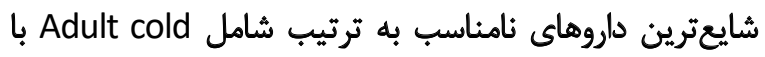

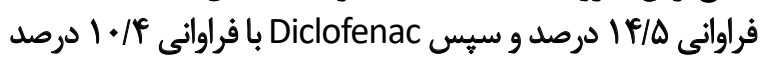
Glibenclamide با فراوانى N9 د درصد بودند

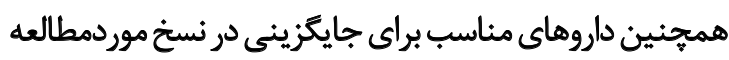

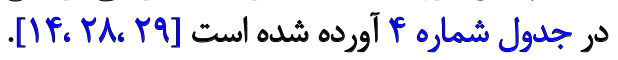

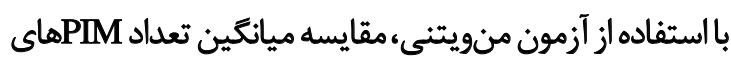



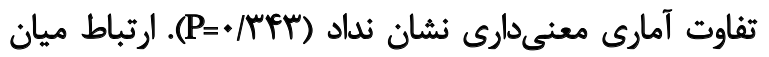

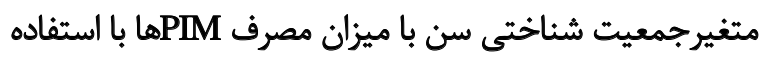

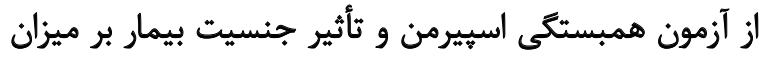

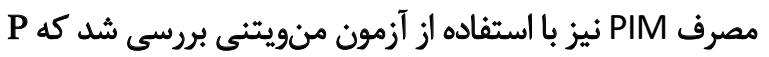

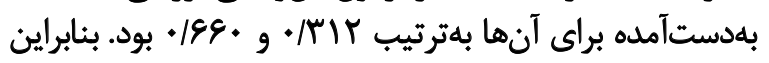

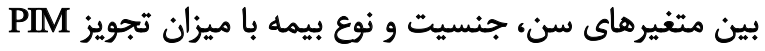

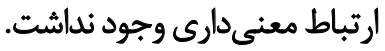

$\stackrel{\leftrightarrow}{\leftrightarrow}$ هدف از اين مطالعها، بررسى وضعيت تجويز داروهاى نامناسب



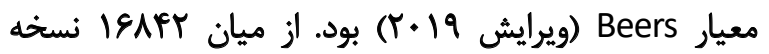

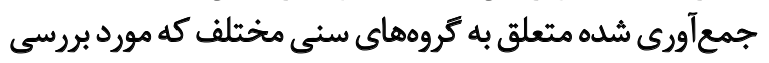


جدول Fأ. داروهاى مناسب جايكزين براى PIM جويزى موجود در نسخ موردبررسى

داروهاى مناسب جايكزين

\section{هاي تجويزي}

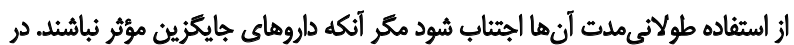

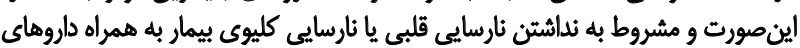

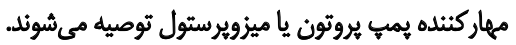

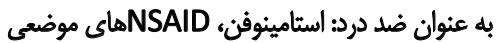

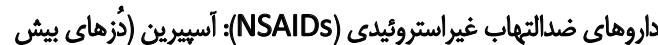

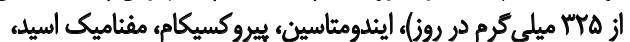

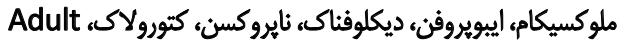
cold

براى تسكين دردهاى فورى خفيف تا متوسط عضلانى: استامينوفن، ايبويروفن، نايبروكسن

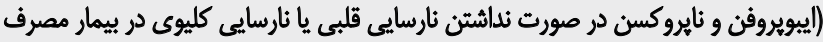

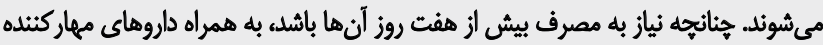

يمبي بروتون توصيل مى نشود).

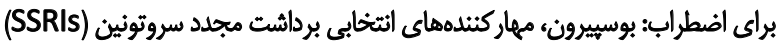

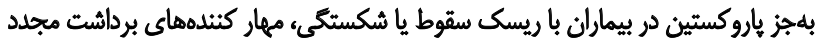
سروتونين نورائ نفرين (SNRIs)

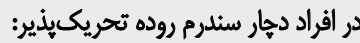

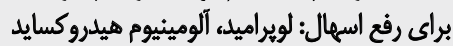

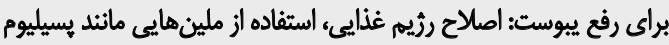

ضداسياسمها: دى سيكلومين، كليدينيوم سى، هيوسين

براي افسردكى:

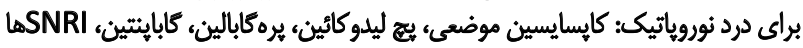

براى تهوع: آنتاكونيستهاى سروتونين مانثد اندانسترون يا كرانيسترون

داروهاى كوارشيى: متوكلويراميد

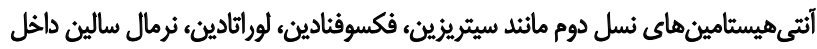

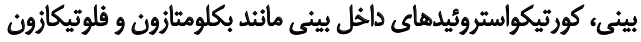

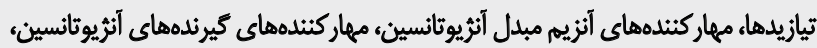

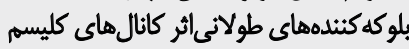

ضلافسردكىها: نورترييتيلين، آمى تريتيتين، ايمىيرامين، دوكسيين

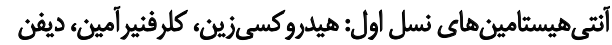

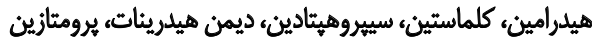

ضد يرفشارى خون: كلوئيدين، هيرازوسين

متفورمين، سولفونيل اورههاى كوتاهاثر مانثد كلى كلازيد يا كلى بييزايد

ترجيحاً روش هاى غيردارويى، مصرف طولانى توصيه نمى شود.

ضدديابت: كلى بئكلاميد

ل ولودويا/كاريىدويا

سلاثيو / هيينوتيكها: زولييدم

ل اموتريثرين، لوتيراستام

ضدتشنجه: فنوباريينال

انجام شد [צr]، نسخ يزشكان عمومى مورد بررسى قرار كرفت.

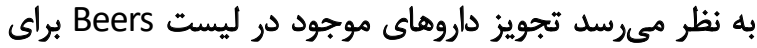

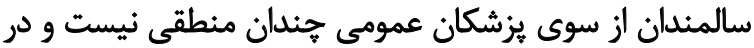

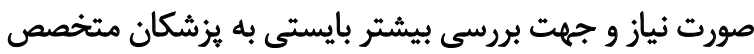

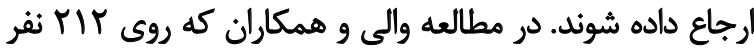

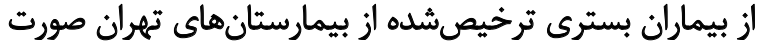

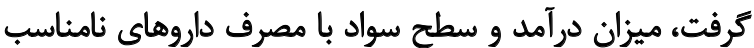

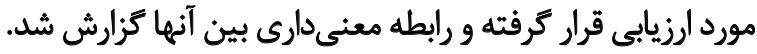

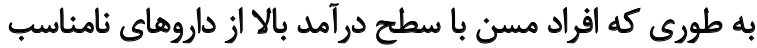

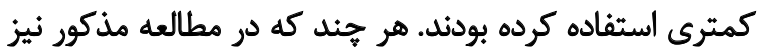

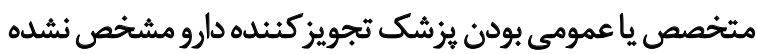

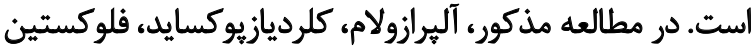

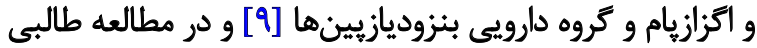

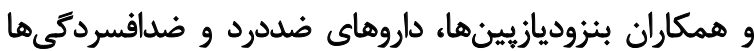

Adult cold، Diclofenac، Glib- سالمندان عبارت بودند از Diphenhydramine g enclamide، Ketorolac مشابه انجاميافته در ايران مى توان به مطالعه حيدرى و همكاران

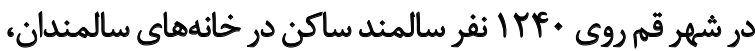

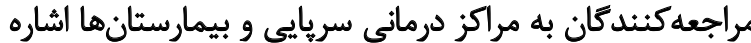

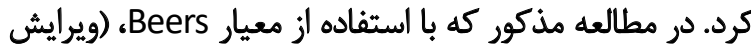

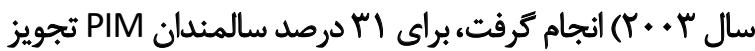

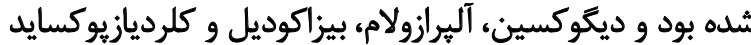

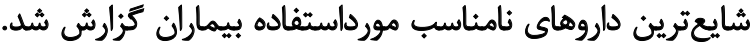

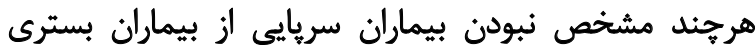

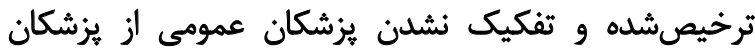

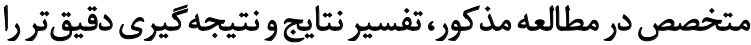

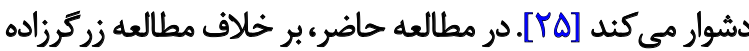

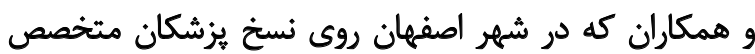




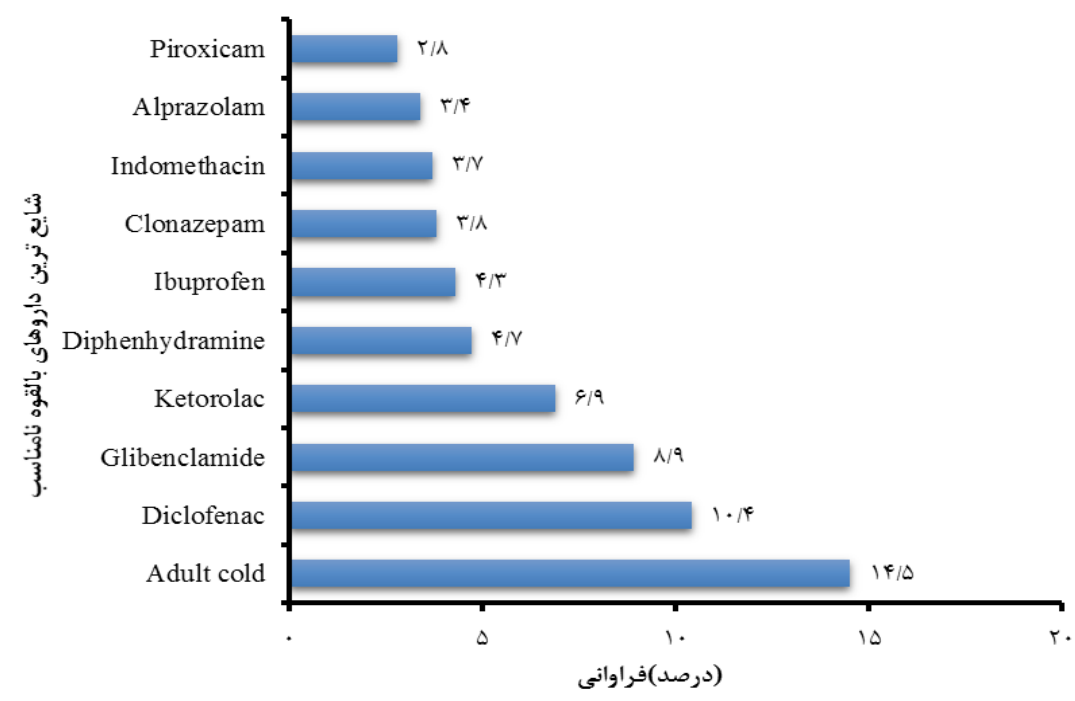

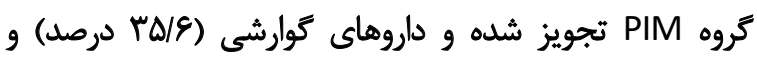

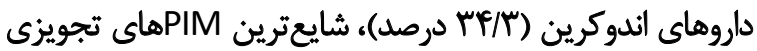

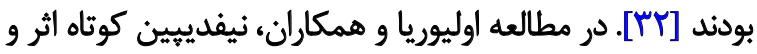

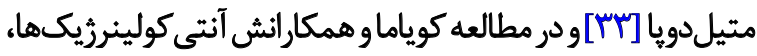

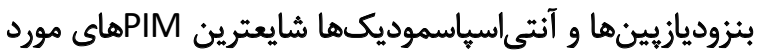

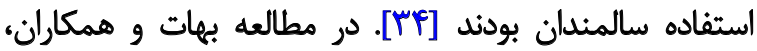



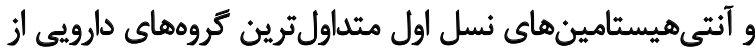

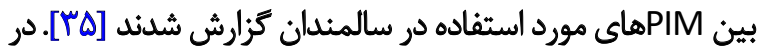

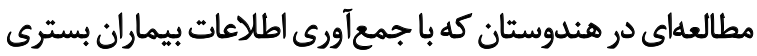

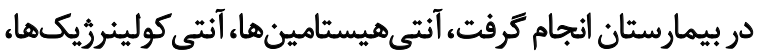

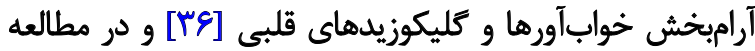

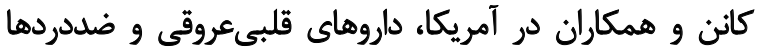

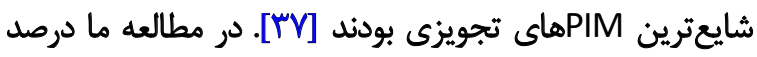

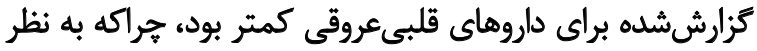

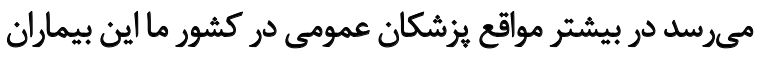



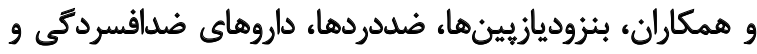

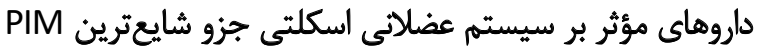

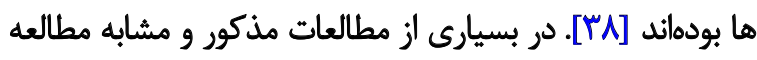

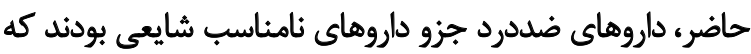

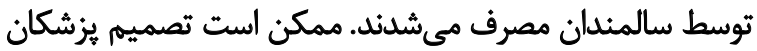

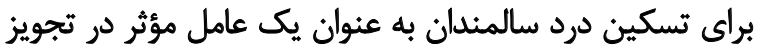

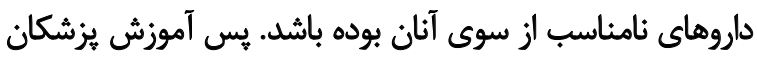

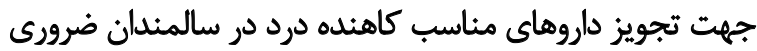

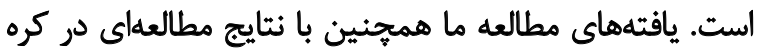

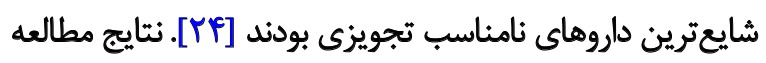

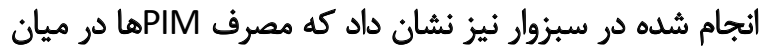





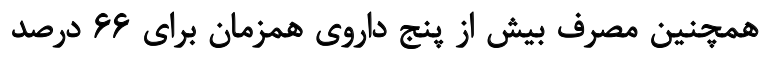



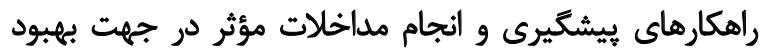

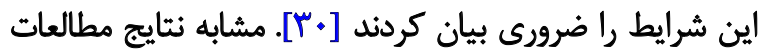

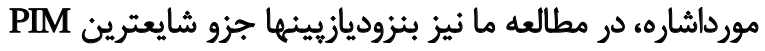

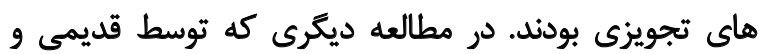

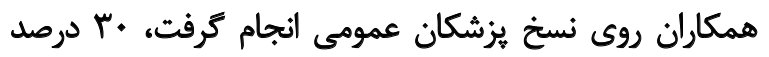

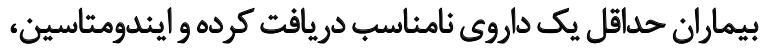

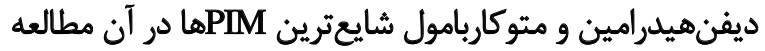

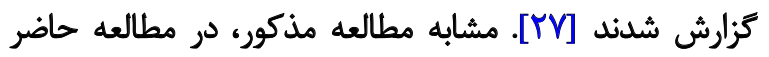

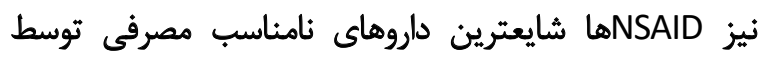

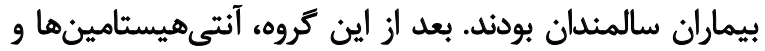

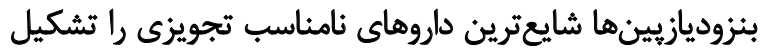

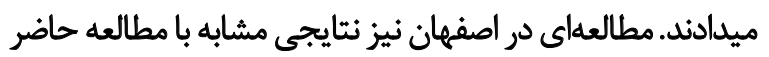

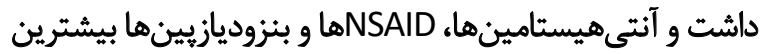

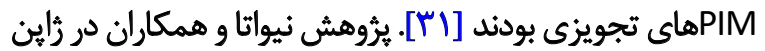

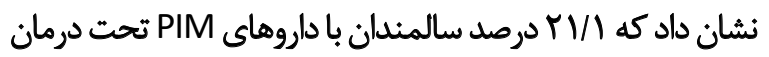

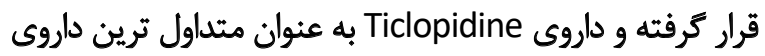
نامناسب تجويزشده براى آنان بود [1ها] نتايج مطالعه الحواسى و همكاران در عربستان سعودى نيز

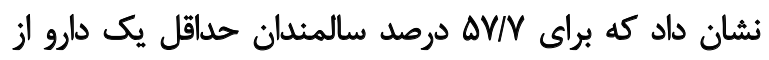


با توجه به دادهاي اين مطالعه، مي توان نتيجه كرفت كه حدود

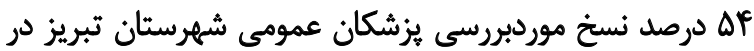

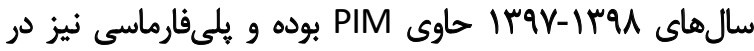

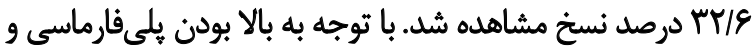

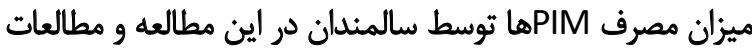

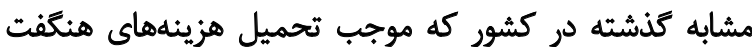

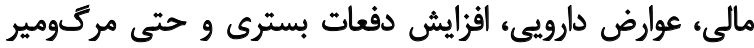

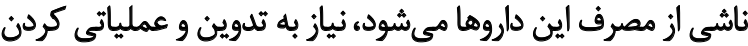



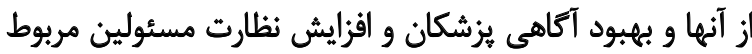



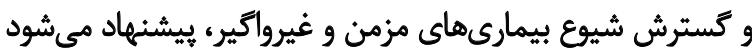

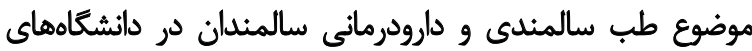

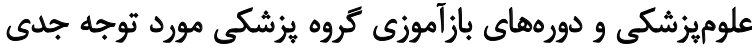

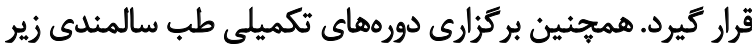

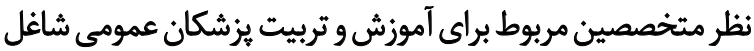

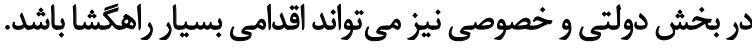
از محدوديتهاي مطالعه حاضر آن بود كه فقط به استفاده از

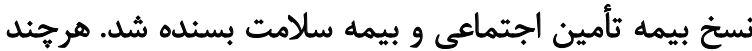

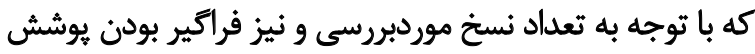

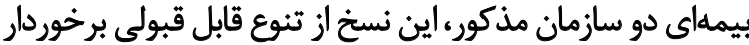

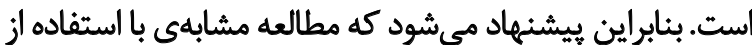

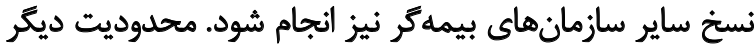

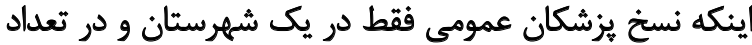

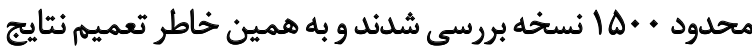

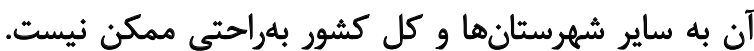

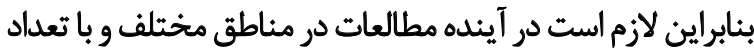

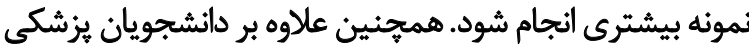

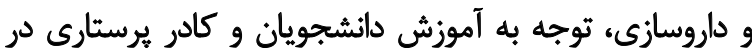

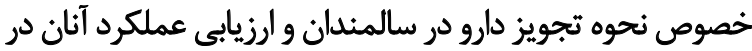
مطالعات آينده ضرورى و بسيار مفيد خواهد بورئ بود.

مالاحظات اخلاقي

\section{يبيروى أز اصول اخلاق يُوهش}

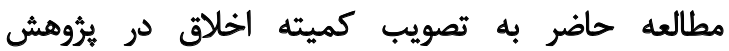
دانشعاه علوميزشكى تبريز رسيده است (كد: IR.TBZMED.

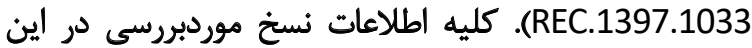
مطالعه به صورت محرمانه است.

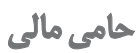

اين مقاله بركرفته از هاياننامه دكتراى عمومى داروسازى

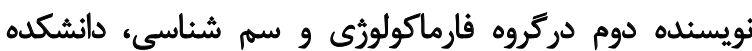

$$
\text { داروسازى، دانشعاه علوم يزشكى دوم تبريز، تبريز است. }
$$

جنوبى همخوانى دارد.در مطالعه مذكور داروهاي آنتى هيستامينى



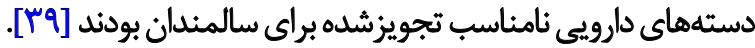
موضوع ديكرى كه بايد به آن توجه كرد آن است كه سياستهاى

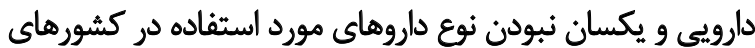
مختلف يكى از علل ايجاد تفاوت در نتئايج مطالعات مختلف درون است.

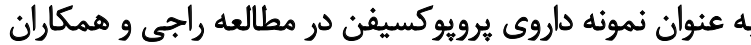

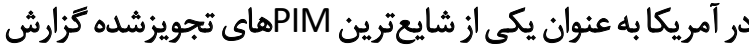

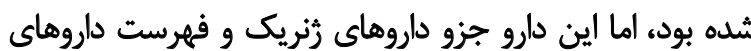

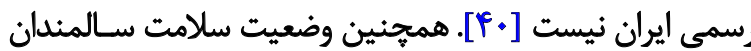

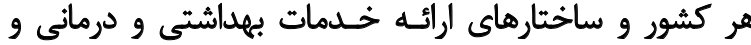



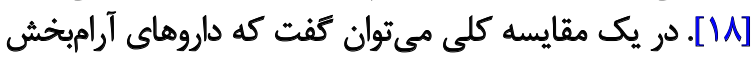

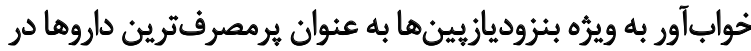

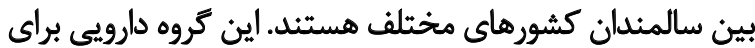

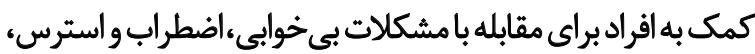



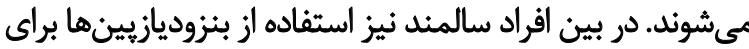

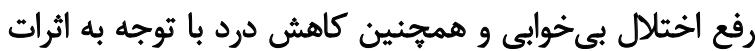

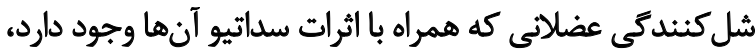

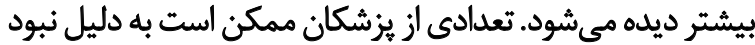

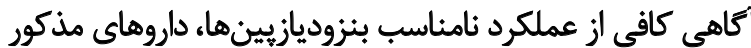

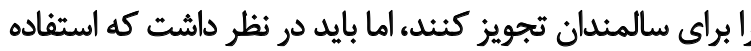

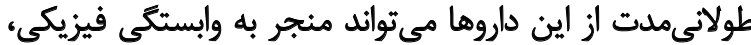

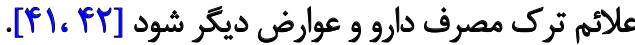

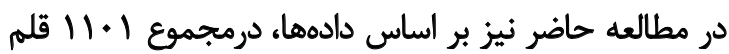

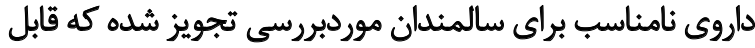

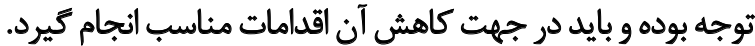

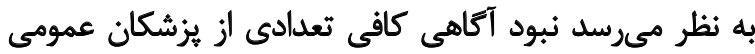

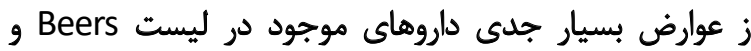

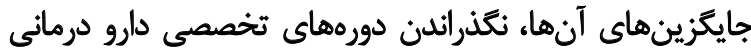

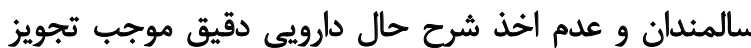

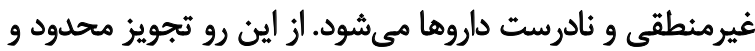

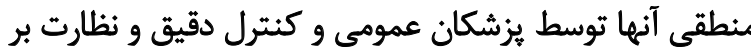

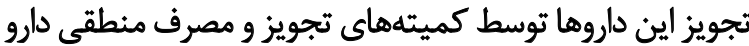

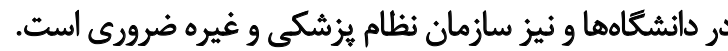
در مطالعه حاضر، وجود ارتباط بين متغيرهاى سن، جنس

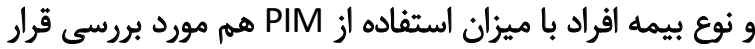

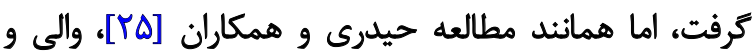

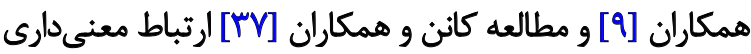
بين استفاده از PIM و اين سه متغير مشاهده نشاند.

نتيجليَيرىنهايى 


$$
\text { مشاركت ثويسند انمان }
$$

تمام نويسندكان در طراحي، اجراو نكارش هما همه بخشهاى ئروهش حاضر مشاركت داشتهاند.

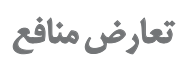

بنابر اظهارنويسندكان اين مقاله تعارض منافع ندارد.

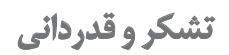

نويسندكان مقاله از مسئولين محترم سازمان بيمه سلامت

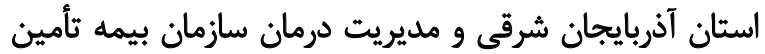

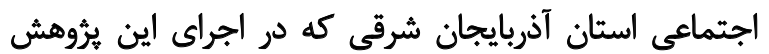
همكارى كردند صميمانه سياسكزارى مى كنيند. 


\section{References}

[1] United Nations. World population ageing 2017 [Internet]. 2017 [Updated 2017]. Available from: https://www.un.org/en/ development/desa/population/publications/pdf/ageing/ WPA2017_Highlights.pdf

[2] Ghasemi S, Keshavarz Mohammadi N, Mohammadi Shahboulaghi F, Ramezankhani A, Mehrabi Y. [Physical health status and frailty index in community dwelling older adults in Tehran (Persian)]. Salmand: Iranian Journal of Ageing. 2019; 13(5):652-65. [DOI:10.32598/SIJA.13.Special-Issue.652]

[3] Karami Matin B, Rezaei S, Alinia S, Shaahmadi F, Kazemi Karyani A. [Ageing in Iran in 1410, a warning to health care system (Persian)]. Journal of Medicine and Cultivation. 2013; 22(2):9-18. http://www.tebvatazkiye.ir/article_79449.html

[4] Vali L, Pourreza A, Ahmadi B, Akbari Sari A. [Quality assessment tools and Management for medication prescription in elderly (Persian)]. Hospital. 2013; 11(4):25-34. http://jhosp.tums. ac.ir/article-1-11-fa.html

[5] Esmaeili Shahmirzadi S, Shojaeizadeh D, Azam K, Salehi L, Tol A, Moradian Sorkhkolaei M. [The impact of chronic diseases on the quality of life among the elderly people in the East of Tehran (Persian)]. Payavard Salamat. 2012; 6(3):225-35. http:/ / payavard. tums.ac.ir/article-1-30-en.html

[6] Peiman H, Yaghoubi M, Seyed Mohammadi A, Delpishe A. [Prevalence of chronic diseases in the elderly in Ilam (Persian)]. Salmand: Iranian Journal of Ageing. 2012; 6(4):7-13. http://salmandj.uswr.ac.ir/article-1-470-en.htm

[7] Woo EK, Han Ch, Jo SA, Park MK, Kim S, Kim E, et al. Morbidity and related factors among elderly people in South Korea: Results from the Ansan Geriatric (AGE) cohort study. BMC Public Health. 2007; 7:10. [DOI:10.1186/1471-2458-7-10] [PMID] [PMCID]

[8] Ghicavii V, Podgurschi L, Pogonea I, Rakovski T. Peculiarities of using drugs in the elderly. The Moldovan Medical Journal. 2017; 60(4):20-4. https://zenodo.org/record/1106597

[9] Vali L, Pourreza A, Rahimi Foroushani A, Akbari Sari A, Honarmand Pharm H. An investigation on inappropriate medication applied among elderly patients. World Applied Sciences Journal. 2012; 16(6):819-25. https://www.idosi.org/wasj/wasj16(6)12/7. pdf

[10] Dianati M, Shojaegharebag GA, Mesdaghinia A, Taghadosi M, Shenasa F, Taiebi A, et al. [Polypharmacy and its related factors among the elderly population in Kashan, Iran during 2011-2012 (Persian)]. Feyz. 2015; 18(6):578-84. http:// feyz.kaums.ac.ir/article-1-2473-en.html

[11] Skov J, Bladbjerg EM, Sidelmann J, Vamosi M, Jespersen J. Plenty of pills: Polypharmacy prevails in patients of a Danish anticoagulant clinic. European Journal of Clinical Pharmacology. 2011; 67(11):1169-74. [DOI:10.1007/s00228-011-1045-0] [PMID]

[12] Lai HY, Hwang SJ, Chen YC, Chen T], Lin MH, Chen LK. Prevalence of the prescribing of potentially inappropriate medications at ambulatory care visits by elderly patients covered by the Taiwanese National Health Insurance program. Clinical Therapeutics. 2009; 31(8):1859-70. [DOI:10.1016/j.clinthera.2009.08.023]

[13] Mirzaei M, Kavosi Z, Vali L, Mahmoodi L. [Study of inappropriate medication prescribed to elderly hospitalized patients using the screening tool to alert doctors to right treatment (Per- sian)]. Salmand: Iranian Journal of Ageing. 2016; 11(2):280-9. [DOI:10.21859/sija-1102280]

[14] The 2019 American Geriatrics Society Beers Criteria ${ }^{\circledR}$ Update Expert Panel. American Geriatrics Society 2019 Updated AGS Beers Criteria ${ }^{\circledR}$ for potentially inappropriate medication use in older adults. Journal of the American Geriatrics Society. 2019; 67(4):674-94. [DOI:10.1111/jgs.15767] [PMID]

[15] Niwata S, Yamada Y, Ikegami N. Prevalence of inappropriate medication using Beers criteria in Japanese long-term care facilities. BMC Geriatrics. 2006; 6:1. [DOI:10.1186/1471-2318-6-1] [PMID] [PMCID]

[16] Fialová D, Topinková E, Gambassi G, Finne-Soveri H, Jónsson PV, Carpenter I, et al. Potentially inappropriate medication use among elderly home care patients in Europe. JAMA. 2005; 293(11):1348-58. [DOI:10.1001/jama.293.11.1348] [PMID]

[17] Cahir C, Bennett K, Teljeur C, Fahey T. Potentially inappropriate prescribing and adverse health outcomes in community dwelling older patients. British Journal of Clinical Pharmacology. 2014; 77(1):201-10. [DOI:10.1111/bcp.12161] [PMID] [PMCID]

[18] Vali L, Pour Reza A, Rahimi Foroushani A, Ahmadi B, Akbari Kamrani AA. [Analysis of inappropriate medication use in older adults discharged from hospitals affiliated with Tehran University of Medical Sciences (TUMS) using the beers criteria in 2010 (Persian)]. Salmand: Iranian Journal of Ageing. 2011; 6(3):56-65. http://salmandj.uswr.ac.ir/article-1-438-en.htm

[19] Van Heerden JA, Burger JR, Gerber JJ. Inappropriate medicine prescribing in older South Africans: A cross-sectional analysis of medicine claims data. South African Medical Journal. 2016 106(10):1010-6. [DOI:10.7196/SAMJ.2016.v106i10.10627] [PMID]

[20] Khamis S, Abdi AM, Uzan A, Basgut B. Applying Beers Criteria for elderly patients to assess rational drug use at a university hospital in Northern Cyprus. Journal of Pharmacy \& BioAllied Sciences. 2019; 11(2):133-41. [DOI:10.4103/jpbs.JPBS_208_18] [PMID] [PMCID]

[21] Rajeev A, Paul G, George S, Vijayakumar P. The study on use of potentially inappropriate medications in elderly patients presenting to a tertiary care hospital in Kerala. International Journal of Scientific Research. 2018; 7(2):12-3. https:/ /www.worldwidejournals.com/international-journal-of-scientific-research-(IJSR)/ fileview.php?val=February_2018_1517579843_292.pdf

[22] Jhaveri BN, Patel TK, Barvaliya MJ, Tripathi Ch. Utilization of potentially inappropriate medications in elderly patients in a tertiary care teaching hospital in India. Perspectives in Clinical Research. 2014; 5(4):184-9. [DOI:10.4103/2229-3485.140562] [PMID] [PMCID]

[23] Momin TG, Pandya RN, Rana DA, Patel VJ. Use of potentially inappropriate medications in hospitalized elderly at a teaching hospital: A comparison between Beers 2003 and 2012 criteria. Indian Journal of Pharmacology. 2013; 45(6):603-7. [DOI:10.4103/0253-7613.121372] [PMID] [PMCID]

[24] Talebi Taher M, Moosavi SAJ, Taherian S, Barati M. [Surveying the inappropriate drug adminstrution using Beers criteria in elderly patients at the internal medicine ward of Rasoul-e-Akram Hospital of Tehran in 2012 (Persian)]. Journal of Arak University of Medical Sciences. 2014; 17(2):33-9. http://jams.arakmu.ac.ir/ article-1-2697-en.html

[25] Heidari S, Aliabadi A, Naebi A, Khoramirad A. [Frequency of use of potentially inappropriate medications and its associated factors in elderly people in Qom city, 2012, Iran (Persian)]. Qom 
University of Medical Sciences Journal. 2014; 8(4):44-52. http:// journal.muq.ac.ir/article-1-407-en.html

[26] Zargarzadeh AH, Sadeghi K, Mirmoghtadaei P. Prescribing of Potentially Inappropriate Medications to Elderly People by Medical Specialists in Isfahan, Iran. Iranian Journal of Pharmaceutical Sciences. 2008; 4(4):241-6. http://www.ijps.ir/article_2069.html

[27] Ghadimi H, Esmaily HM, Wahlstrom R. General practitioners' prescribing patterns for the elderly in a province of Iran. Pharmacoepidemiology \& Drug Safety. 2011; 20(5):482-7. [DOI:10.1002/ pds.2106]

[28] Hanlon JT, Semla TP, Schmader KE. Alternative medications for medications in the use of high-risk medications in the elderly and potentially harmful drug-disease interactions in the elderly quality measures. Journal of the American Geriatrics Society. 2015; 63(12):e8-18. [DOI:10.1111/igs.13807] [PMID] [PMCID]

[29] Therapeutic Research Center. Potentially harmful drugs in the elderly: Beers list [Internet]. 2019 [Updated 2019 March]. Available from: https://www.pharmacyquality.com/wp-content/ uploads/2019/05/Beers-List-350301.pdf

[30] Torabi B, Mohammadi M, Aboozade Gatabi Kh, Ghanbari Moghaddam A. [Study of prescribing unsuitable drugs and polypharmacy in the elderly admitted to the Vasei Hospital of Sabzevar, 2017 (Persian)]. Journal of Gerontology. 2018; 3(2):48-55. [DOI:10.29252/joge.3.1.48]

[31] Azoulay L, Zargarzadeh A, Salahshouri Z, Oraichi D, Bérard A. Inappropriate medication prescribing in community-dwelling elderly people living in Iran. European Journal of Clinical Pharmacology. 2005; 61(12):913-9. [DOI:10.1007/s00228-005-0036-4] [PMID]

[32] Alhawassi TM, Alatawi W, Alwhaibi M. Prevalence of potentially inappropriate medications use among older adults and risk factors using the 2015 American Geriatrics Society Beers criteria. BMC Geriatrics. 2019; 19:154. [DOI:10.1186/s12877-019-1168-1] [PMID] [PMCID]

[33] Oliveira MG, Amorim WW, de Jesus SR, Rodrigues VA, Passos LC. Factors associated with potentially inappropriate medication use by the elderly in the Brazilian primary care setting. International Journal of Clinical Pharmacy. 2012; 34(4):626-32. [DOI:10.1007/s11096-012-9656-9] [PMID]

[34] Koyama A, Steinman M, Ensrud K, Hillier TA, Yaffe K. Tenyear trajectory of potentially inappropriate medications in very old women: Importance of cognitive status. Journal of the American Geriatrics Society. 2013; 61(2):258-63. [DOI:10.1111/jgs.12093] [PMID] [PMCID]

[35] Bhatt AN, Paul SS, Krishnamoorthy S, Baby BT, Mathew A, Nair BR. Potentially inappropriate medications prescribed for older persons: A study from two teaching hospitals in Southern India. Journal of Family Community Medicine. 2019; 26(3):187-92. [PMID] [PMCID]

[36] Zaveri HG, Mansuri SM, Patel VJ. Use of potentially inappropriate medicines in elderly: A prospective study in medicine out-patient department of a tertiary care teaching hospital. Indian Journal of Pharmacology. 2010; 42(2):95-8. [PMID] [PMCID]

[37] Cannon KT, Choi MM, Zuniga MA. Potentially inappropriate medication use in elderly patients receiving home health care: A retrospective data analysis. The American Journal of Geriatric Pharmacotherapy. 2006; 4(2):134-43. [DOI:10.1016/j.amjopharm.2006.06.010] [PMID]
[38] Pugh MJV, Fincke BG, Bierman AS, Chang BH, Rosen AK Cunningham FE, et al. Potentially inappropriate prescribing in elderly veterans: Are we using the wrong drug, wrong dose, or wrong duration? Journal of the American Geriatrics Society. 2005; 53(8):1282-9. [DOI:10.1111/j.1532-5415.2005.53402.x] [PMID]

[39] Nam YS, Han JS, Kim JY, Bae WK, Lee K. Prescription of potentially inappropriate medication in Korean older adults based on 2012 Beers Criteria: A cross-sectional population based study. BMC Geriatrics. 2016; 16:118. [DOI:10.1186/s12877-016-0285-3] [PMID] [PMCID]

[40] Raji MA, Ostir GV, Markides KS, Espino DV, Goodwin JS. Potentially inappropriate medication use by elderly Mexican Americans. Annals of Pharmacotherapy. 2003; 37(9):1197-202. [DOI:10.1345/aph.1C480] [PMID]

[41] Windle A, Elliot E, Duszynski K, Moore V. Benzodiazepine prescribing in elderly Australian general practice patients. Australian and New Zealand Journal of Public Health. 2007; 31(4):379-81. [DOI:10.1111/j.1753-6405.2007.00091.x]

[42] Guina J, Merrill B. Benzodiazepines I: Upping the care on downers: The evidence of risks, benefits and alternatives. Journal of Clinical Medicine. 2018; 7(2):17. [DOI:10.3390/jcm7020017] [PMID] [PMCID] 